\title{
Maturidade, desempenho académico, capacidade de raciocínio e estatuto sócio- económico em crianças de Lisboa entre os 6 e os 13 anos de idade
}

\author{
Carlos Barrigas \& Isabel Fragoso \\ Faculdade de Motricidade Humana, Portugal
}

\begin{abstract}
Resumo
O presente artigo pretende avaliar a associação entre a maturidade, o desempenho académico (DA), a capacidade de raciocínio (CR) e o estatuto sócio-económico (ESE), numa amostra de 792 crianças (394 rapazes e 398 raparigas), com idades entre os 6 e os 13 anos. A maturidade foi calculada com recurso à idade óssea (Tanner et al., 2001) e o DA foi obtido através de inquérito aos professores, resultados em testes de aferição e níveis alcançados no $2 .^{\circ}$ período lectivo. O ESE foi calculado através da Escala de Graffar (Graffar, 1958) e a CR foi obtida através da aplicação do teste das Matrizes Progressivas Coloridas de Raven (Raven, 1963). Os dados foram tratados com o programa SPSS 17.0 ( $p \leq .05)$. Verificámos que o DA estava directamente relacionado com o ESE, que a CR dos rapazes era superior à das raparigas, que não existiam diferenças entre o DA dos rapazes e das raparigas nas diferentes classes de ESE, que os rapazes mais maturos obtinham resultados académicos superiores aos alcançados pelos menos maturos, que a maturidade estava associada ao DA e que essa associação estava, na generalidade das situações, dependente da associação entre o ESE e o DA.
\end{abstract}

Palavras-chave Maturidade; Desempenho académico; Capacidade de raciocínio; Estatuto sócio-económico

\section{Introdução}

O desempenho académico e a necessidade de melhorar a qualidade das aprendizagens têm criado um contexto de investigação que, nos últimos 
60 anos, se tem revelado como uma das áreas de maior produção científica, em todo o mundo.

O facto de o desempenho escolar resultar da confluência de um número significativamente elevado de factores contribuiu para que este tema pudesse ser analisado numa grande multiplicidade de abordagens, que conseguimos agrupar em torno de três perspectivas de investigação. A primeira perspectiva reúne questões de âmbito sócio-cultural e inclui estudos sobre o estatuto sócio-económico (Coleman et al., 1966; Chall, 1996; Payne \& Biddle, 1999; Thomas \& Stockton, 2009), o grau de literacia da família (Weinstein, 1998; Pierce \& Brisk, 2002; Connelly-Weida, 2007), a participação da família na vida escolar (Epstein, 1995; Osterling, 2001; Barrigas, Vieira, Fragoso, Ferreira, \& Vargas, 2004), a influência dos professores (Ashton, 1996; Greenwald, Hedges, \& Laine, 1996; Fetler, 1999), ou a influência cultural (McKenna, Kear, \& Ellsworth, 1995; Mazzeo, Allen, \& Kline, 1995; Diamond \& Onwuegbuzie, 2001). A segunda perspectiva reúne aspectos de índole psicossocial e abrange estudos na área da inteligência (Jensen, 1998; Frey \& Detterman, 2004; Rohde \& Thompson, 2006), da personalidade (Kifer, 1975; Stewart \& Louisa, 2006; Amin \& Chowdhury, 2006) e da auto-estima (Byrne, 1996; Senos, 1997; Mann, Hosman, Schaalma, \& De Vries, 2004; ElAnzi, 2005; Barrigas, Fragoso, \& Vieira, 2007, 2009). A terceira perspectiva envolve questões de carácter biológico ou biossocial e estudos sobre a velocidade de processamento da informação (Kail, 1992; Eaton \& Ritchot, 1995; Kail \& Miller, 2006; Kail \& Ferrer, 2007), a velocidade de condução do influxo nervoso (Cerella \& Hale, 1994; Kail, 1991), a memória de trabalho (Dempster, 1981; Carpenter, Just, \& Shell, 1990; Kail, 1992; Gathercole \& Baddeley, 1993; Fry \& Hale, 1996), a concentração de hormonas sexuais (Petersen, 1976; Waber, Mann, Merola, \& Moylan, 1985) e a organização do sistema nervoso central (Henry, 1994; Ridderinkhof \& Van der Molen, 1997; Hámori, 2002).

A investigação iniciada na década de 1950, nas áreas da Psicologia Genética, da Sociologia da Educação e da Psicossociologia, tornou evidente a relação entre o desempenho académico e o ESE das famílias e das populações. Os estudos efectuados nestas áreas mostram que as crianças pertencentes a grupos sócio-culturais mais débeis têm níveis de desempenho escolar inferiores (Coleman et al., 1966; Haycock, 2001; Pellino, 2009). As 
causas para os fracos resultados académicos descritas na literatura são numerosas e encontram-se, frequentemente, associadas ao envolvimento social das crianças (envolvimento familiar e comportamentos de aprendizagem) e à qualidade da educação que recebem (formação e atitudes dos professores).

Outra linha de investigação decorrente do aumento da utilização da psicometria, iniciada no princípio da década de 1970, tem vindo a estudar a associação entre a aprendizagem e os testes de avaliação de capacidades intelectuais, particularmente entre os testes de inteligência e as capacidades intelectuais preditoras da aprendizagem académica (Parker \& Benedict, 2002; Watkins, Lei, \& Canivez, 2007). A ideia de que as escalas de 'Ql' e os testes que avaliam o factor ' $g$ ' são os melhores e mais poderosos preditores do desempenho académico (Te Nijenhuis, Tolboom, \& Bleichrodt, 2004) influenciou o modo de encarar o sucesso escolar e transformou a inteligência num factor fundamental para o contexto educativo. Os resultados da investigação efectuada neste âmbito têm apontado para uma correlação moderada entre os testes utilizados para medir a inteligência e algumas variáveis académicas, tais como os resultados escolares ou o número de anos de escolarização dos sujeitos (Naglieri, 1996; Watkins et al., 2007; Lemos, Almeida, Guisande, \& Primi, 2008). Com efeito, vários estudos têm apontado para uma associação entre as habilidades cognitivas e o rendimento escolar nas disciplinas cujo conteúdo curricular se aproxima do conteúdo dos itens utilizados nestas provas de avaliação cognitiva (Sternberg, Grigorenko, \& Bundy, 2001; Kuncel, Hezlett, \& Ones, 2004). No entanto, e como alguns investigadores referem, esta associação tende a diminuir à medida que a escolaridade avança, abrindo a possibilidade de outras variáveis do aluno, do professor ou do contexto de ensino-aprendizagem irem, progressivamente, assumindo maior relevância no rendimento escolar (Barca, Brenella, Canosa, \& Enriquez, 1999; Rosário \& Almeida, 1999).

Uma outra linha de investigação, ainda, tem procurado associar o desempenho académico ao desenvolvimento biológico. O processo de evolução biológica (maturação), ou, mais especificamente, a distância percorrida em direcção ao estado final adulto (maturidade) (Tanner, Healy, Goldstein, \& Cameron, 2001) tem sido frequentemente adiantado como factor de diferenciação e barreira à apreensão dos conteúdos escolares. A influência 
da maturidade no desempenho académico tem sido sugerida ao longo dos últimos 50 anos por muitos autores (Martinez, Garcia, \& Montoro, 1993; Fonseca, 1999; Fragoso et al., 2001; Watkins et al., 2007). Halfter (1962) parece ter sido um dos primeiros investigadores a concluir que a idade cronológica era um mau indicador da performance cognitiva, sugerindo, em alternativa, a utilização de um indicador a que chamou idade funcional. No início da década de 1970, Hull (1970) concluiu que a maturidade, avaliada com base na idade de entrada na escola, era um bom preditor de sucesso educativo. Conscientes da diferença entre as idades biológica e cronológica e acreditando na associação entre maturação biológica e maturação psicológica, Tanner (1962) e Kail e Hall (1999) defendem que as crianças mais maturas são cognitivamente mais eficientes. A disseminação desta ideia pela classe docente é evidente nas afirmações de Crahay (1996), ao concluir que, para $46 \%$ dos professores, a evolução psicológica das crianças é essencialmente um problema de maturação fisiológica. Martinez et al. (1993), Fonseca (1999) e Fragoso et al. (2001) suportam esta ideia, sugerindo que o atraso na maturação do sistema nervoso pode condicionar a capacidade para apreender os estímulos que são proporcionados à criança.

No entanto, e apesar de ser geralmente aceite que os resultados académicos dependem, entre outros factores, da maturidade (Salisbury, Rees, \& Gorard, 1999), apenas encontrámos dois artigos que, de forma objectiva, procuraram associar a maturidade ao desempenho de tarefas cognitivas. Verificámos, ainda, que estes dois artigos apresentavam amostras distintas, utilizavam metodologias de avaliação da maturidade pouco precisas e, talvez por isso, revelavam alguma falta de consistência nos resultados obtidos. O primeiro estudo, realizado por Waber et al. (1985) numa amostra de 145 crianças (78 raparigas e 67 rapazes), com idades entre os 11 e os 19 anos, utilizou as características sexuais secundárias como metodologia de avaliação da maturidade e concluiu que não existiam diferenças na performance em tarefas cognitivas entre crianças avançadas e atrasadas maturacionalmente. O segundo estudo, realizado por Sappington e Topolski (2005) numa amostra de 468 estudantes universitários de ambos os sexos, utilizou a data de início da puberdade, registada retrospectivamente por autoavaliação, e concluiu que a maturidade precoce estava, entre outros factores, associada com a obtenção de resultados mais elevados em matemática. 
A variedade das publicações e a dispersão das perspectivas de análise têm dificultado a percepção do insucesso académico como um fenómeno multidimensional e, simultaneamente, pessoal, já que, apesar de insinuar uma etiologia diversa, o insucesso escolar se anuncia e se materializa num sujeito concreto. Assim, e atendendo ao facto de a generalidade dos estudos sobre o insucesso académico não considerar a globalidade da relação entre alguns factores sociais (estatuto sócio-económico), psicossociais (capacidade de raciocínio e auto-estima) e biológicos (maturidade) e, particularmente, por acharmos que a maturidade, entendida como o nível de progressão do indivíduo para o seu estado maturo ou adulto (Malina, Bouchard, \& Bar-Or, 2004), não tem sido objectivamente ponderada nas inúmeras investigações efectuadas em torno dos resultados escolares dos alunos, propusemo-nos estudar a associação entre o desempenho académico, a capacidade de raciocínio, o estatuto sócio-económico e a maturidade.

\section{Metodologia}

\section{Participantes}

O estudo recaiu sobre uma amostra de 792 crianças, 394 rapazes e 398 raparigas, estudantes de 8 escolas de Lisboa, com idades compreendidas entre os 6 e os 13 anos, que nunca tinham sido retidos.

\section{Instrumentos}

A maturidade foi calculada com recurso ao método da idade óssea TW3 (Tanner et al., 2001), enquanto o desempenho académico foi obtido através de questionário aos professores, testes de aferição e resultados da avaliação dos alunos no $2^{\circ}$ período de avaliação; o estatuto sócio-económico foi calculado através da Escala de Notação Social de Graffar (Graffar, 1958) e a capacidade de raciocínio foi obtida através da aplicação do teste das Matrizes Progressivas Coloridas de Raven - MPCR (Raven, 1963; Raven, Raven, \& Court, 2003).

\section{Procedimentos}

$\mathrm{O}$ indicador de maturidade foi obtido com recurso à diferença entre a idade cronológica e a idade óssea. Por falta de recursos para a utilização do 
mesmo procedimento de recolha dos resultados do desempenho académico nos alunos dos $1 .^{\circ}$ e $2 .^{\circ}$ Ciclos do Ensino Básico (CEB), optámos por utilizar a média entre a avaliação atribuída por questionário aos professores nas áreas disciplinares de Língua Portuguesa (LP), Matemática (M) e Estudo do Meio $(E M)$ e os resultados conseguidos pelos alunos nos testes de aferição nas mesmas áreas disciplinares no $1 .^{\circ} \mathrm{CEB}$ e a média dos resultados alcançados no segundo momento de avaliação (Páscoa) nas disciplinas de LP, M e Ciências da Natureza (CN) no 2. ${ }^{\circ}$ CEB. Os testes de aferição utilizados no $1 .^{\circ}$ CEB foram realizados por ano e disciplina, por uma equipa de professores profissionalizados com larga experiência de leccionação, e aplicados no final do segundo período lectivo.

Por termos tido poucos sujeitos com resultados escolares muito baixos em ambos os ciclos de escolaridade, optámos por reunir a totalidade dos alunos com desempenho académico abaixo da média (resultados inferiores a $49 \%)$ no escalão 1) que denominamos resultados não satisfatórios, tendo os restantes sido agrupados nos escalões: 2) resultados satisfatórios (50\% a $74 \%)$; 3) resultados bons (75\% a $84 \%)$; e 4 ) resultados muito bons ( $85 \%$ a $100 \%)$. Normalizámos os resultados escolares por ano, idade e disciplina, através da técnica score $Z$, para podermos comparar idades e sexos e anular o habitual aumento de exigência dos professores e a consequente diminuição do aproveitamento escolar dos alunos, à medida que estes se vão aproximando da transição de ciclo de escolaridade. No cálculo do estatuto ESE, utilizámos como indicadores o nível escolar e a profissão dos pais e o local e tipo de habitação, que classificámos segundo a metodologia proposta por Graffar em 1958. Dividimos a distribuição em tercis e agrupámos os participantes em 3 classes que denominámos: 1) baixa; 2) média; e 3) alta.

O teste MPCR foi aplicado colectivamente e os resultados foram tratados segundo a proposta de aferição para a população portuguesa (Simões, 2000). Estes resultados deram origem a 5 classes, correspondendo a classe 1 aos alunos com resultados inferiores ao percentil 20 e as restantes classes (2 a 5) aos resultados inferiores ao percentil 40, 60, 80 e 100, respectivamente. Os dados foram tratados com o programa SPSS 17.0 para Windows. Para o cálculo das correlações, utilizámos o coeficiente de correlação de Pearson e, para as diferenças, utilizámos o teste $t$, a análise da variância (ANOVA) e a análise da co-variância (ANCOVA), com a capacidade 
de raciocínio, o ESE e a capacidade de raciocínio e o ESE como co-variáveis. Como é habitual em estudos desta natureza, a significância estatística foi estabelecida em 0.05

O protocolo experimental cumpriu as exigências da Declaração de Helsínquia e foi previamente aprovado pela Comissão de Ética da Faculdade de Motricidade Humana. Os objectivos e os procedimentos foram explicados aos pais e apenas foram incluídos na amostra os participantes cujos tutores legais assinaram um consentimento informado.

\section{Resultados}

No quadro 1, apresentamos os valores médios e desvios-padrão encontrados em cada variável por sexo e para a totalidade da amostra.

\section{Quadro 1 - Valores médios e desvio-padrão, por sexo e total, nas variáveis em estudo}

\begin{tabular}{ccccccc}
\hline & & \multicolumn{6}{c}{ Valores médios e desvio-padrão } \\
\cline { 3 - 7 } & $\mathbf{N}$ & Idade decimal & Idade óssea & $\begin{array}{c}\text { Capac. } \\
\text { raciocínio }\end{array}$ & $\begin{array}{c}\text { Desemp. } \\
\text { Académico }\end{array}$ & ESE \\
\hline Rapazes & 394 & $9.22(1.7)$ & $8.93(1.8)$ & $63.62(29.4)$ & $3.56(0.8)$ & $8.41(3.2)$ \\
Raparigas & 368 & $9.08(1.7)$ & $8.94(2.3)$ & $57.10(30.1)$ & $3.62(0.9)$ & $8.63(3.4)$ \\
Total & 792 & $9.16(1.7)$ & $8.93(2.0)$ & $60.47(29.9)$ & $3.59(0.9)$ & $8.52(3.3)$ \\
\hline
\end{tabular}

Aplicámos o teste $\mathrm{t}$ de pares aos resultados globais apresentados no Quadro 1 e verificámos que existiam diferenças entre os valores obtidos nas idades decimal e óssea, quer quando considerámos a totalidade da amostra $(p=.001)$ quer quando dividimos a amostra em função do sexo ( $p=.001$ e $p=.019$, respectivamente para rapazes e raparigas), e nos valores obtidos na capacidade de raciocínio pelos rapazes e pelas raparigas $(p=.006)$.

Na figura 1 apresentamos os valores do desempenho académico, por sexo e por classe de estatuto sócio-económico. Como podemos visualizar, o valor médio do desempenho académico aumentou, em ambos os sexos, à medida que a classe de ESE aumentou também. 


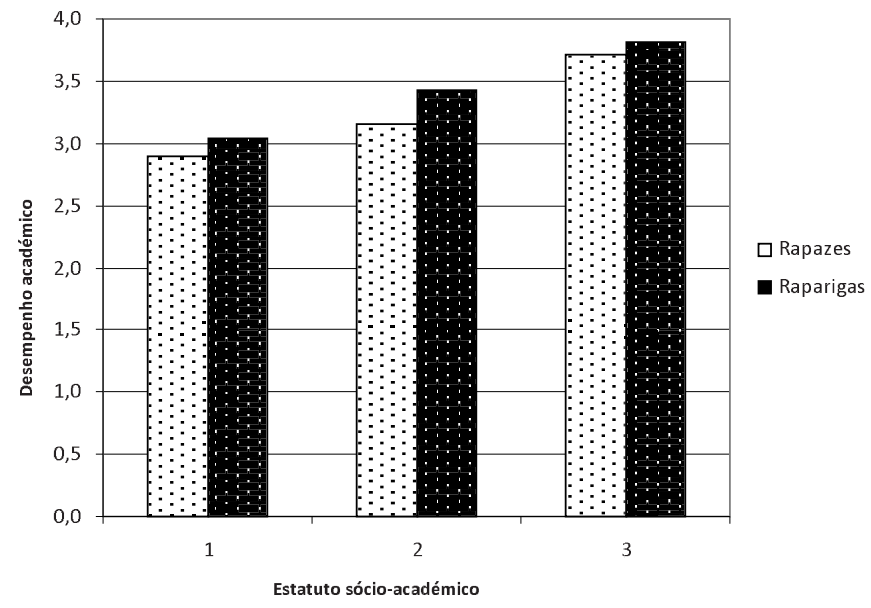

Figura 1 - Valores de desempenho académico (média dos resultados atribuídos pelos professores e obtidos pelos alunos nas fichas de avaliação nas áreas disciplinares de LP, M e EM/CN) em ambos os sexos em função das classes de estatuto sócio-económico (1- baixo, 2médio e 3- alto)

Utilizando o coeficiente de correlação de Pearson, obtivemos valores de correlação significativos $(p=.001)$ entre ambos os sexos, em todos os níveis de ESE. Quando utilizámos a ANOVA, para comparar o desempenho académico de rapazes e raparigas, nas três classes de ESE (1 - baixo, 2 médio e 3 - alto), verificámos a existência de diferenças entre as classes 1 e $3(p=.001)$ e 2 e $3(p=.001)$ nos rapazes, e entre as classes 1 e $2(p=.031), 1$ e $3(p=.001)$ e 2 e $3(p=.007)$ nas raparigas. Utilizando o teste $t$ de pares, verificámos que não existiam diferenças entre o desempenho académico dos rapazes e das raparigas, quer na globalidade da amostra quer nas diferentes classes de ESE.

Na figura 2 apresentamos os valores de desempenho académico em ambos os sexos, em função das classes definidas para a capacidade de raciocínio. Como pode ser visualizado, verificámos que o desempenho académico aumenta com o aumento da capacidade de raciocínio, em ambos 
os sexos. Utilizando o coeficiente de correlação de Pearson, obtivemos valores de correlação significativos $(p=.001)$ em ambos os sexos e em todas as classes de raciocínio.

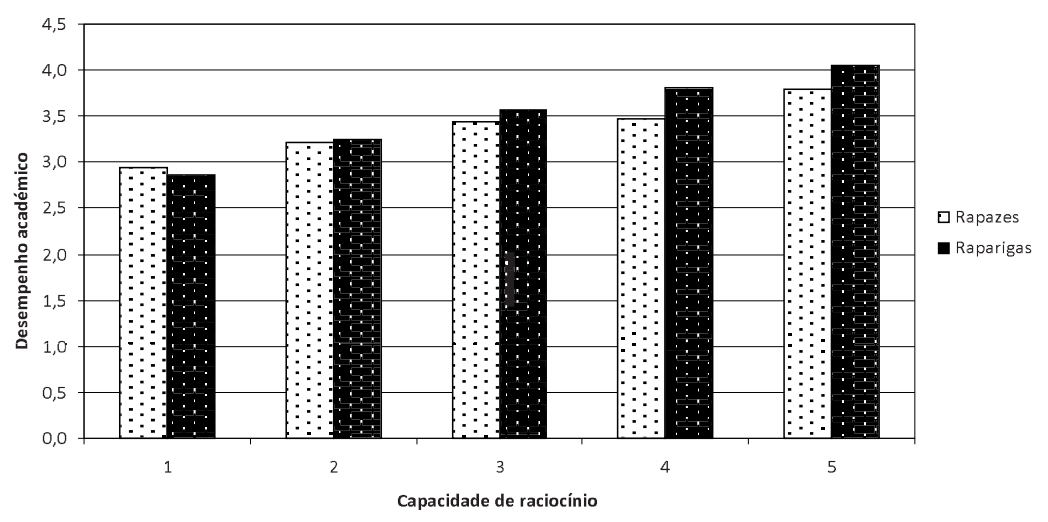

Figura 2 - Valores de desempenho académico (média dos resultados atribuídos pelos professores e obtidos pelos alunos nas fichas de avaliação nas áreas disciplinares de LP, M e EM/CN) em ambos os sexos em função dos níveis de capacidade de raciocínio (1 - baixo, 2 médio baixo, 3 - médio, 4 - médio alto e 5 - alto)

Utilizando o teste $\mathrm{t}$ de pares, verificámos que, embora tendo obtido valores inferiores na capacidade de raciocínio, as raparigas apresentavam valores de desempenho académico superiores aos dos rapazes, na média das disciplinas $(p=.001)$ e em LP $(p=.035)$. Utilizando a ANOVA, verificámos que existiam diferenças nos resultados do desempenho académico global entre os grupos 1 e $5(p=.001)$ e 2 e $5(p=.001)$ nos rapazes, e entre os grupos 1 e $3(0.001), 1$ e $4(p=.001), 1$ e $5(0.001), 2$ e $4(P=.005), 2$ e $5(p=.001)$ e 3 e $5(p=.005)$ nas raparigas.

$\mathrm{Na}$ figura 3 apresentamos os valores obtidos na maturidade, em ambos os sexos, em função do estatuto sócio-económico. 


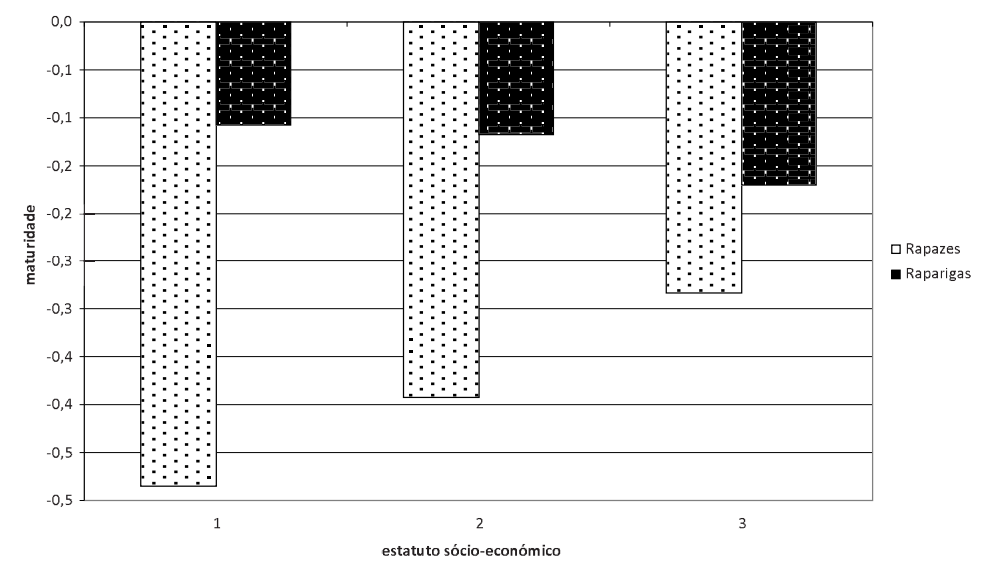

Figura 3 - Valores de maturidade (diferença entre a idade óssea e a idade decimal) em ambos os sexos em função do estatuto sócioeconómico (1 - baixo, 2 - médio e 3 - alto)

Como podemos ver na figura 3, quando analisámos a maturidade em função do ESE verificámos que os rapazes apresentam um comportamento oposto ao das raparigas. Com efeito, a diferença entre a idade óssea e a idade cronológica diminui à medida que o ESE aumenta nos rapazes e, inversamente, aumenta à medida que o ESE aumenta nas raparigas. Utilizando o teste t de pares, verificámos que apenas existiam diferenças na maturidade entre rapazes e raparigas na classe 1 de ESE $(p=.047)$.

A figura 4 apresenta-nos os resultados da maturidade (diferença entre a idade óssea e a idade decimal) em função da capacidade de raciocínio. $A$ ANOVA mostrou-nos que apenas existiam diferenças na maturidade entre os rapazes dos grupos 4 e $5(p=.033)$ e que não existiam diferenças entre rapazes e raparigas em cada escalão de raciocínio. $O$ resultado do coeficiente de correlação de Pearson revelou a ausência de correlação entre a maturidade e a capacidade de raciocínio, quer nos rapazes quer nas raparigas. 


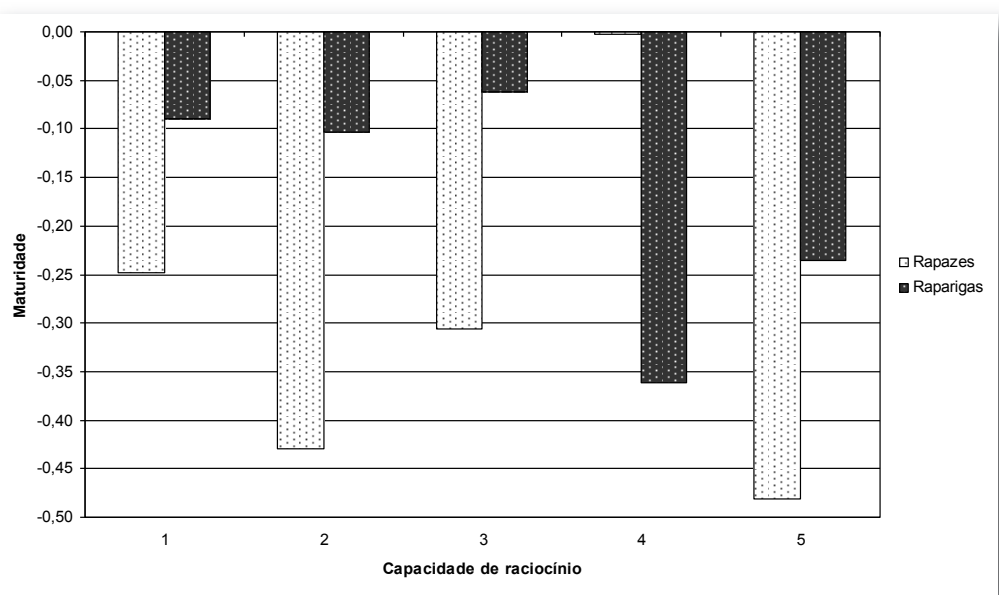

Figura 4 - Valores de maturidade (diferença entre a idade óssea e a idade decimal) em ambos os sexos em função dos níveis de capacidade de raciocínio (1 - baixo, 2 - médio baixo, 3 - médio, 4 médio alto e 5 - alto)

Utilizando os tercis da diferença entre a idade óssea e a idade cronológica, dividimos a amostra em 3 níveis maturacionais (1 - atrasados, 2 - normais e 3 - avançados) e procurámos verificar se existiam diferenças nos valores normalizados do desempenho académico, entre estes mesmos grupos, em cada sexo.

Como podemos observar no quadro 2, utilizando a ANOVA, verificámos a existência de diferenças entre os grupos 1 e $2(p=.030)$ em M e 1 e $3(p=.035)$ em EM/CN nos rapazes, e entre os grupos 1 e $3(p=.029)$ e 2 e $3(p=.009)$ em M e 2 e $3(p=.027)$ em EM nas raparigas. Utilizando a ANCOVA com a capacidade de raciocínio como covariável, verificámos a existência de diferenças entre os grupos 1 e $2(p=.049)$ e 1 e $3(p=.036)$ em LP, 1 e $2(p=.014)$ e 1 e $3(p=.014)$ em $M$, e 1 e $3(p=.004)$ em EM/CN nos rapazes, e entre os grupos 1 e $3(p=.039)$ e 2 e $3(p=.010)$ em $M$, e 1 e 3 $(p=.052)$ e 2 e $3(p=.017)$ em $E M / C N$ nas raparigas. Utilizando a ANCOVA com o ESE como covariável verificámos a existência de diferenças entre os grupos 1 e $3(p=.021)$ em LP, 1 e $2(p=.036)$ e 1 e $3(p=.002)$ em $M$, e 1 e $3(p=.008)$ em EM/CN nos rapazes, e os grupos 2 e 3 ( $p=.032)$ em $M$ nas raparigas. 
Quadro 2 - Diferenças (ANOVA e ANCOVA com a capacidade de raciocínio, o ESE e a capacidade de raciocínio e o ESE como covariáveis) no desempenho académico nas disciplinas de Língua

Portuguesa (LP), Matemática (M) e Estudo do Meio/Ciências da Natureza (EM/CN) entre os grupos de maturação 1) atrasados, 2) normais e 3) avançados

\begin{tabular}{|c|c|c|c|c|c|c|c|c|c|c|c|c|}
\hline & & \multicolumn{5}{|c|}{ ANOVA } & \multicolumn{2}{|c|}{ ANCOVA (CR) } & \multicolumn{2}{|c|}{ ANCOVA (ESE) } & \multicolumn{2}{|c|}{$\begin{array}{c}\text { ANCOVA (CR + } \\
\text { ESE) }\end{array}$} \\
\hline & & & $\mathbf{N}$ & Média & sd & Comp (p) & Média aj. & Comp (p) & Média aj. & Comp (p) & Média aj & Comp (p) \\
\hline \multirow{10}{*}{ 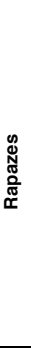 } & \multirow{3}{*}{ ב } & 1 & 126 & 2.55 & 0.8 & & 2.52 & $1-2$ & 2.52 & & 2.52 & \\
\hline & & 2 & 125 & 2.78 & 0.9 & & 2.75 & (.049) & 2.71 & $1-3$ & 2.70 & $1-3$ \\
\hline & & 3 & 109 & 2.76 & 0.9 & & 2.77 & (.036) & 2.91 & & 2.79 & \\
\hline & \multirow{4}{*}{$\sum_{\Sigma}^{\pi}$} & 1 & 126 & 2.61 & 0.9 & & 2.61 & $1-2$ & 2.59 & $1-2$ & 2.63 & \\
\hline & & 2 & 125 & 2.92 & 0.9 & $1-2$ & 2.91 & (.014) & 2.83 & $(.036)$ & 2.86 & $1-3$ \\
\hline & & & & & & $(.030)$ & & $1-3$ & & $1-3$ & & $(.014)$ \\
\hline & & 3 & 109 & 2.88 & 0.9 & & 2.92 & $(.014)$ & 3.14 & $(.002)$ & 2.94 & \\
\hline & \multirow{3}{*}{$\begin{array}{l}\text { Z } \\
\sum_{\text {i }}\end{array}$} & 1 & 126 & 2.72 & 0.9 & \multirow{3}{*}{$\begin{array}{c}1-3 \\
(.035)\end{array}$} & 2.69 & \multirow{3}{*}{$\begin{array}{c}1-3 \\
(.004)\end{array}$} & 2.72 & \multirow{3}{*}{$\begin{array}{c}1-3 \\
(.008)\end{array}$} & 2.70 & $1-3$ \\
\hline & & 2 & 126 & 2.96 & 0.9 & & 2.91 & & 2.92 & & 2.85 & $(.002)$ \\
\hline & & 3 & 109 & 3.02 & 0.9 & & 3.03 & & 3.18 & & 3.01 & \\
\hline \multirow{10}{*}{ 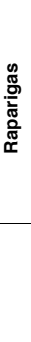 } & \multirow{4}{*}{9} & 1 & 109 & 2.89 & 0.9 & & 2.86 & & 2.82 & & 2.86 & \\
\hline & & 2 & 109 & 2.95 & 0.9 & & 2.87 & & 2.86 & & 2.89 & \\
\hline & & 3 & 126 & 2.71 & 0.9 & & 2.73 & & 2.85 & & 2.76 & \\
\hline & & 1 & 109 & 2.86 & 0.9 & $1-3$ & 2.83 & $1-3$ & 2.77 & & 2.83 & \\
\hline & \multirow{2}{*}{$\tilde{\Sigma}^{\pi}$} & 2 & 109 & 2.92 & 1.0 & (.029) & 2.90 & (.039) & 2.86 & $2-3$ & 2.97 & $2-3$ \\
\hline & & 3 & 126 & 252 & 1.0 & $\begin{array}{l}2-3 \\
(209)\end{array}$ & 260 & $\begin{array}{l}2-3 \\
(010)\end{array}$ & 254 & & 263 & $(.012)$ \\
\hline & \multirow{4}{*}{$\begin{array}{l}\text { Z } \\
\sum_{\text {Z }}\end{array}$} & 1 & 110 & 2.97 & 0.9 & \multirow{4}{*}{$\begin{array}{c}2-3 \\
(.027)\end{array}$} & 2.91 & $1-3$ & 2.88 & & 2.91 & \multirow{4}{*}{$\begin{array}{c}2-3 \\
(.022)\end{array}$} \\
\hline & & 2 & 109 & 3.01 & 0.9 & & 2.96 & (.052) & 2.92 & & 2.97 & \\
\hline & & & & & & & & $2-3$ & & & & \\
\hline & & 3 & 126 & 2.71 & 0.8 & & 2.69 & $(.017)$ & 2.72 & & 2.70 & \\
\hline
\end{tabular}

Utilizando, simultaneamente, a capacidade de raciocínio e o ESE como covariáveis na ANCOVA, verificámos que nos rapazes passavam a existir apenas diferenças entre os grupos 1 e 3 em LP $(p=.035)$ e M $(p=.014)$ e se mantinham as diferenças já anteriormente verificadas entre os grupos 1 e $3(p=.002)$ em $E M / C N$, enquanto nas raparigas apenas se verificavam diferenças entre os grupos 2 e 3 em M (P=.012) e em EM/CN ( $p=.022)$.

\section{Discussão e conclusões}

O presente trabalho teve como objectivo estudar a relação entre o desempenho académico, o ESE, a capacidade de raciocínio e a maturidade, 
em 792 alunos (394 rapazes e 398 raparigas), de 8 escolas de Lisboa, com idades entre os 6 e os 13 anos e que nunca tinham sido retidos.

A diminuição dos valores do desempenho académico em função do ESE, observados no nosso estudo, realça a associação entre o ESE e os resultados escolares e está de acordo com os resultados encontrados na generalidade dos estudos realizados nesta área. Porém, quando analisámos os resultados do desempenho académico em função do ESE (figura 1) e os cruzámos com a informação referente à evolução da maturidade em função do ESE (figura 3), ficámos com a ideia de que as raparigas pertencentes ao ESE mais baixo, e portanto mais maturas, obtinham resultados significativamente piores no desempenho académico. Se a relação observada neste sexo entre o desempenho académico e o ESE era esperada (Sammons, 1995; Gillborn \& Gipps, 1996), a relação entre o ESE e a maturidade, contrária à relação encontrada nos rapazes, apresenta alguma singularidade.

Os resultados encontrados na amostra feminina do nosso estudo contrariaram as conclusões dos estudos de Guedes e Guedes (1991) e de Malina et al. (2004), que concluíram que as raparigas educadas em meios sociais favorecidos alcançavam a puberdade mais cedo do que as educadas em meios sócio-económicos desfavorecidos. Contudo, estes resultados poderão traduzir os efeitos da discriminação social baseada no sexo, reportados por Bogin (1991), Moreira, Fragoso, e Júnior (2004) e Marques (2008) e consubstanciados na forma de pressão social exercida mais intensamente sobre as raparigas dos grupos sociais mais desfavorecidos que poderá, na nossa perspectiva, justificar o avanço maturacional encontrado nas raparigas pertencentes às classes de ESE mais baixo, impelindo-as a atingir um estado biológico mais evoluído para, provavelmente, poderem assumir a sua função social de suporte familiar e reprodução, mais precocemente do que os rapazes.

Quando utilizámos a ANCOVA e o ESE como covariável (quadro 2), verificámos que, para além das diferenças já observadas, os rapazes passavam a apresentar diferenças de desempenho em LP e M entre os grupos atrasado e avançado e as raparigas deixavam de apresentar diferenças entre os grupos atrasado e avançado em $\mathrm{M}$ e normal e avançado em EM/CN, o que sugere, uma vez mais, a influência fundamental do ESE 
sobre o desempenho académico, em particular nas raparigas. Assim, diríamos que, apesar de a pobreza constituir um factor de risco para o insucesso na escola ou na vida (Kaiser \& Delaney, 1996; Leroy \& Symes, 2001), no caso das raparigas essa influência é determinante, sobrepondo-se às diferenças de desempenho que parecem resultar da maturidade.

A utilização do teste MPCR procurou identificar uma associação entre as habilidades cognitivas e o rendimento escolar, nas disciplinas cujo conteúdo curricular se aproxima do conteúdo dos itens utilizados nestas provas de avaliação cognitiva, tal como tem sido apresentado em diversos estudos (Kuncel et al., 2004; Sternberg et al., 2001). A relação entre a capacidade de raciocínio e o desempenho académico encontrada no nosso estudo mostra que a capacidade de raciocínio, avaliada através do teste MPCR, segue o mesmo padrão de associação encontrado entre as provas habitualmente utilizadas para medir a inteligência (WISC, WAIS, etc.) e o desempenho académico, ou seja, mostra que as crianças que obtêm bons resultados nos testes de inteligência obtêm, também, bons resultados escolares. O facto de a nossa amostra apenas abranger crianças até aos 13 anos não nos permitiu, contudo, verificar se esta relação diminui com o avançar da escolaridade obrigatória. Num futuro próximo, esperamos ter oportunidade de realizar um estudo que inclua jovens com idades mais avançadas, de modo a podermos verificar se outras variáveis do processo de ensino-aprendizagem diluem esta relação e, progressivamente, vão adquirindo maior relevância no desempenho escolar.

Quando agrupámos a amostra em função da capacidade de raciocínio, verificámos que as raparigas conseguiram melhores resultados no desempenho académico nos grupos 2, 3, 4 e 5, apesar de terem alcançado valores de capacidade de raciocínio inferiores aos dos rapazes. As características não verbais do teste MPCR, a utilização diferenciada de estratégias mentais (Fennema, Carpenter, Jacobs, Franke, \& Levi, 1998), o menor interesse e a menor confiança das raparigas nas suas capacidades na matemática (Catsambis, 1994), ou a maior capacidade dos rapazes para o cálculo matemático (Eccles, Wigfield, Harold, \& Blumenfield, 1993; Barlow, 2006), podem ter contribuído para que estes obtivessem melhores resultados na prova de capacidade de raciocínio. 
Os melhores resultados académicos obtidos pelas raparigas, no nosso estudo, mostram, no entanto, que a superioridade dos rapazes na capacidade de raciocínio não foi, por si só, suficiente para que estes conseguissem também melhores resultados escolares. Com efeito, ficámos com a ideia de que a habitual superioridade das raparigas no domínio da linguagem (Undheim \& Nordvik, 1992; Burman, Bitan, \& Booth, 2008) ou na capacidade de concentração (Hunt, 1985), as opções e atitudes femininas na escolha de carreiras (Catsambis, 1994), a superioridade das raparigas para a composição reflexiva (Salisbury et al., 1999), ou o encorajamento e a valorização dos professores desta mesma capacidade de descrição na escrita (Stobart, Elwood, \& Quinlan, 1992), factores não avaliados no nosso estudo, poderão ter tido um efeito fundamental na obtenção de melhores resultados académicos globais e, muito particularmente, na obtenção de resultados superiores em LP. Uma vez mais, esperamos ter oportunidade de confirmar esta suposição, posteriormente.

Ainda neste âmbito, gostaríamos de fazer referência aos resultados do quadro 2. Quando a variável capacidade de raciocínio é utilizada como covariável (ANCOVA), os rapazes passam a apresentar diferenças de desempenho na LP, entre grupos de maturidade (quadro 2). Esta alteração de comportamento parece mostrar que a capacidade de raciocínio atenua as diferenças de desempenho resultantes das diferenças maturacionais nos rapazes, o que levanta a possibilidade de a capacidade de raciocínio ser uma variável importante no desempenho académico dos rapazes.

As diferenças de desempenho académico encontradas entre os grupos de maturação (quadro 2) parecem indicar que o nível maturacional influencia o desempenho académico global. Com efeito, quando dividimos a amostra em três grupos maturacionais, verificámos que os rapazes com maturidade normal ou avançada apresentavam resultados superiores aos seus pares com maturidade atrasada, e que as raparigas com maturidade atrasada e normal apresentavam resultados superiores às avançadas nas áreas disciplinares $\mathrm{M}$ e $\mathrm{EM} / \mathrm{CN}$.

A área disciplinar de LP parece, no entanto, não ser influenciada pela maturação, já que, depois de analisados os resultados do desempenho nesta disciplina, por grupos de maturidade, verificámos que não existiam diferenças entre os valores obtidos pelos três grupos de maturidade, quer nos rapazes quer nas raparigas. 
Verificámos, também, que os resultados do desempenho aumentam, em todas as disciplinas, nos rapazes, do grupo de maturação atrasado para o avançado, quando se divide o desempenho por níveis de maturação e quando, na ANCOVA, se utilizam como covariáveis a capacidade de raciocínio e o ESE isoladamente ou a capacidade de raciocínio e o ESE conjuntamente, dando, uma vez mais, a ideia de que, apesar destas covariáveis exercerem uma influência importante no desempenho académico, a maturação não deixa, por isso, de apresentar diferenças significativas entre grupos extremos, após a sua remoção. Confirmamos, deste modo, os resultados de Sappington e Topolski (2005) e as sugestões menos objectivas, mas fundamentais, dadas por muitos autores, desde 1962 (Halfter, 1962; Tanner, 1962; Hull, 1970), que levaram Crahay, em 1996, a difundir, pela classe docente, a ideia de que a evolução psicológica das crianças é, essencialmente, um problema de maturação fisiológica, e Martinez et al. (1993), Fonseca (1999) e Fragoso et al. (2001) a estudarem este tema mais objectivamente.

\section{Nota Final}

O presente estudo sugere uma associação entre a maturidade e o desempenho académico. Convém, no entanto, recordar que a maturidade é influenciada por subtis alterações em factores tão diversos como a alimentação, a saúde ou a qualidade e quantidade da exposição solar, fortemente dependentes do ESE, e que estas alterações determinam o tempo e o momento de ocorrência de pequenas variações bioquímicas que, por sua vez, determinam a maturidade. Torna-se, portanto, extremamente difícil separar os efeitos da maturidade e do estatuto sócio-económico no desempenho académico. Estamos, contudo, convencidos de que os resultados obtidos no nosso estudo poderão ter sido condicionados pela idade baixa e, portanto, pela diminuta amplitude maturacional das crianças que constituíram a nossa amostra. Admitimos a possibilidade de os resultados poderem ter outra expressão se a amostra incluísse sujeitos com idades superiores e uma maior amplitude maturacional em cada escalão de idade. 


\section{Referências}

Amin, M. N., \& Chowdhury, M. S. (2006). Personality and students' academic achievement: Interactive effects of conscientiousness and agreeableness on students' performance in principles of economics. Social Behavior \& Personality, 34(4), 381-388.

Ashton, P. T. (1996). Improving the preparation of teachers. Educational Researcher, 25(9), 21-22.

Barca, A., Brenella, J. C., Canosa, S., \& Enriquez, A. G. (1999). Estratégias e enfoques de aprendizaje, contextos familiares y rendimiento académico en el alumnado de educación secundária: Indicadores para un análisis causal. Revista GalegoPortuguesa de Psicoloxia e Educación, 4(3), 229-269.

Barlow, J. (2006). Approach to school affects how girls compare with boys in Math. Disponível em http://news.illinois.edu/NEWS/06/0220math divide.html

Barrigas, C., Fragoso, I., \& Vieira, F. (2007). Is maturation an important measure for the study of self-esteem? In I. Fragoso, F. Carnide \& F. Vieira (Eds.), The 3rd International Symposium on Measurement, Analysis and Modelling of Human Functions (pp. 233-238). Cruz Quebrada: Faculdade de Motricidade Humana.

Barrigas, C., Fragoso, I., \& Vieira, F. (2009). Self-esteem, weight status and maturity in Portuguese school children. In P. Hume \& A. Stewart (Eds.), Kinanthropometry XI: 2008 Pre-Olympic Congress Anthropometry Research (pp. 69-77). Auckland, New Zealand: Sport Performance Research Institute.

Barrigas, C., Vieira, F., Fragoso. I., Ferreira, C., \& Vargas, T. (2004). A importância do envolvimento parental na realização dos trabalhos de casa. Quid Novi, 5, 67-78.

Bogin, B. (1991). Patterns of human growth. Cambridge: Cambridge University Press.

Burman, D. D., Bitan, T., \& Booth, J. R. (2008). Sex differences in neural processing of language among children. Neuropsychologia, 46, 1349-1362.

Byrne, B. M. (1996). Academic self-concept: Is structure, measurement and relation to academic achievement. In B. A. Bracken (Ed.), Handbook of self-concept (pp. 287-316). New York: John Wiley \& Sons.

Carpenter, P. A., Just, M. A., \& Shell, P. (1990). What one intelligence test measures: A theoretical account of the processing in the Raven progressive matrices test. Psychological Review, 97(3), 404-431.

Catsambis, S. (1994). The path to Math: Gender and racial-ethnic differences in Mathematics participation from Middle School to High School. Sociology of Education, 67(3), 199-215.

Cerella, J., \& Hale, S. (1994). The rise and fall in information-processing rates over the life span. Acta Psychologica (Amsterdam), 82(2-3), 109-197.

Chall, J. S. (1996). American reading achievement: Should we sorry? Research in the Teaching of English, 30, 303-310.

Coleman, J. S., Campbell, E. Q., Hobson, C. J., McPartland, J., Mood, A. M, Weinfeld, F. D., \& York, R. L. (1966). Equality of Educational Opportunity Report. Washington DC: U.S. Government Printing Office. 
Connelly-Weida, C. (2007). Family literacy concepts for increased K-12 success: A review of the literature. Disponível em http://www.pde. state.pa.us/ able/lib/able/field notes07/fn07flliterature.pdf

Crahay, M. (1996). Podemos lutar contra o insucesso escolar? Lisboa: Instituto Piaget.

Dempster, F. N. (1981). Memory span: Sources of individual and developmental differences. Psychological Bulletin, 89(1), 63-100.

Diamond, P. J., \& Onwuegbuzie, A. J. (2001). Factors associated with reading achievement and attitudes among elementary school-aged students. Research in the Schools, 8, 1-11.

Eaton, W. O., \& Ritchot, K. F. M. (1995). Physical maturation and information processing speed in middle childhood. Developmental Psychology, 31(6), 967-972.

Eccles, J., Wigfield, A., Harold, R. D., \& Blumenfield, P. (1993). Age and gender differences in children's self- and task perceptions during elementary school. Child Development, 64, 830-847.

El-Anzi, F. (2005). Academic achievement and its relation with anxiety, self-esteem, optimism, and pessimism in Kuwaiti students. Social Behaviour and Personality, 33(1), 95-104.

Epstein, J. L. (1995). School/family/community partnerships caring for the children we share. Phi Betta Kappan, 76, 701-711.

Fennema, E., Carpenter, T. P., Jacobs, V. R., Franke, M. L., \& Levi, L. W. (1998). A longitudinal study of gender differences in young children's mathematical thinking. Educational Researcher, 27(5), 6-11.

Fetler, M. (1999). High school staff characteristics and mathematics test results. Education Policy Analysis Archives, 7(9), Disponível em http://epaa.asu.edu/ epaa/v7n9.html.

Fonseca, V. (1999). Insucesso escolar: Abordagem psicopedagógica das dificuldades de aprendizagem ( $2^{\mathrm{a}}$ ed.). Lisboa: Âncora.

Fragoso, I., Vieira, F., Barrigas, C., Oliveira, C., Silva, L., \& Magalhães, M. (2001). Maturação e sucesso escolar. Quid Novi, 1, 24-32.

Frey, M. C., \& Detterman, G. K. (2004). Scholastic assessment or g? The relationship between the scholastic assessment test and general cognitive ability. Psychological Science, 15(6), 373-378.

Fry, A. F., \& Hale, S. (1996). Processing speed, working memory, and fluid intelligence: Evidence for a developmental cascade. Psychological Science, 7(4), 237-241.

Gathercole, S. F., \& Baddeley, A. (1993). Working memory and language. Hillsdale: Erlbaum.

Gillborn, D., \& Gipps, C. (1996). Recent research on the achievements of ethnic minority pupils. London: HMSO.

Graffar, M. (1958). Une méthode de classification d'achantillon de population. Courrier, 6, 455-478.

Greenwald, R., Hedges, L., \& Laine, R. (1996). The effect of school resources on student achievement. Review of Educational Research, 66(3), 361-396. 
Guedes, D. P., \& Guedes, J. P. (1991). Influência do nível socioeconômico e do aspecto racional em variáveis antropométricas e motoras de moças maturadas e não maturadas. Revista Brasileira de Ciência e Movimento, 5, 41-51.

Halfter, I. T. (1962). The comparison of achievement of young and old. National Association of Women Deans and Counselors, 25, 60-67.

Hámori, J. (2002). Plasticity and gender differences of the developing brain. Humanbiologia Budapestinensis, 27, 13-16.

Haycock, K. (2001). Closing the achievement gap. Educational Leadership, 58(6), 6-11.

Henry, L. (1994). The relationship between speech rate and memory span in children. International Journal of Behavioral Development, 17(1), 37-56.

Hull, D. (1970). Maturity as a variable in predicting academic success. Columbia: Missouri University College of Education.

Hunt, E. (1985). Verbal ability. In R. Sternberg (Ed.), Human abilities (pp. 31-58). New York: Freeman \& Co.

Jensen, A. R. (1998). The $g$ factor: The science of mental ability. Westport: Greenwood Publishing.

Kail, R. V. (1991). Developmental change in speed processing during childhood and adolescence. Psychological Bulletin, 109(3), 490-501.

Kail, R. V. (1992). Processing speed, speech rate, and memory. Developmental Psychology, 28(5), 899-904.

Kail, R. V., \& Ferrer, E. (2007). Processing speed in childhood and adolescence: Longitudinal models for examining developmental change. Child Development, 78(6), 1760-1770.

Kail, R. V., \& Hall, L. K. (1999). Sources of developmental change in children's wordproblem performance. Journal of Educational Psychology, 91(4), 660-668.

Kail, R. V., \& Miller, C. A. (2006). Developmental change in processing speed: Domain specificity and stability. Journal of Cognition and Development, 7(1), 119-137.

Kaiser, A., \& Delaney, E. (1996). The effects of poverty on parenting young children. Peabody Journal of Education, 71(4), 66-85.

Kifer, E. (1975). Relationships between academic achievement and personality characteristics: A quasi-longitudinal study. American Educational Research Journal, 12(2), 191-210.

Kuncel, N., Hezlett, S., \& Ones, D. S. (2004). Academic performance, career potencial, creativity and job performance: Can one construct predict them all? Journal of Personality and Social Psychology, 86, 148-161.

Lemos, G., Almeida, L. S., Guisande, M. A., \& Primi, R. (2008). Inteligência e rendimento escolar: Análise da sua relação ao longo da escolaridade. Revista Portuguesa de Educação, 21(1), 83-99.

Leroy, C., \& Symes, B. (2001). Teachers' perspectives on the family backgrounds of children at risk. McGill Journal of Education, 36(1), 45-60.

Malina, R. M., Bouchard, C., \& Bar-Or, O. (2004). Growth, maturation and physical activity ( $2^{\mathrm{a}}$ ed.). Champaign, IL: Human Kinetics. 
Mann, M., Hosman, C. M. H., Schaalma, H. P., \& De Vries, N. K. (2004). Self-esteem in a broad-spectrum approach for mental health promotion. Health Education Research, 19(4), 357-372.

Marques, A. M. (2008). Questões de género. In Ministério do Trabalho e da Solidariedade Social - PETI (Ed.), 10 anos de combate à exploração do trabalho infantil em Portugal (pp. 155-170). Lisboa: Ministério do Trabalho e da Solidariedade Social - PETI.

Martinez, M. P., Garcia, M. C., \& Montoro, J. M. (1993). Dificuldades de aprendizagem. Porto: Porto Editora.

Mazzeo, J., Allen, N. L., \& Kline, D. L. (1995). The 1994 NAEP Trial Assessment. National Center for Educational Statistics. Disponível em http://nces.ed.gov/nationsreportcard/y25sa/tsa94.shtml

McKenna, M., Kear, D., \& Ellsworth, R. (1995). Children's attitudes toward reading: A national survey. Reading Research Quarterly, 30, 934-956.

Moreira, D., Fragoso, I., \& Júnior, A. (2004). Socioeconomic and maturational levels of young samba dancers from Rio de Janeiro. Revista Brasileira de Medicina do Esporte, 10(1), 24-30.

Naglieri, J. A. (1996). An examination of the relationship between intelligence and reading achievement using the MAT-SF and MAST. Journal of Psychoeducational Assessment, 14(1), 65-69.

Osterling, J. P. (2001). Waking the sleeping giant: Engaging and capitalizing on the socio-cultural strengths of the Latino community. Bilingual Research Journal, 25(1/2), 59-88.

Parker, D. R., \& Benedict, K. B. (2002). Assessment and intervention: Promoting successful transitions for college students with ADHD. Assessment for Effective Intervention, 27(3), 3-24.

Payne, K., \& Biddle, B. (1999). Poor school funding, child poverty and mathematics achievement. Educational Researcher, 28(6), 4-13.

Pellino, K. M. (2009). The effects of poverty on teaching and learning. Disponível em http://www.teach-nology.com/tutorials/teaching/ poverty /print.htm

Petersen, A. C. (1976). Physical androgyny and cognitive functioning in adolescence. Developmental Psychology, 12(6), 524-533.

Pierce, M., \& Brisk, M. E. (2002). Sharing the bilingual journey: Situational autobiography in a family literacy context. Bilingual Research Journal, 2(3), 575597.

Raven, J. C. (1963). The advanced progressive matrices. London: H.K. Lewis.

Raven, J., Raven, J. C., \& Court, J. H. (2003). Manual for Raven's progressive matrices and vocabulary scales. Section 1: general overview. San Antonio: Harcourt.

Ridderinkhof, K. R., \& Van der Molen, M. W. (1997). Mental resources, processing speed, and inhibitory control: A developmental perspective. Biological Psychology, 45, 241-261.

Rohde, T. E., \& Thompson, L. A. (2006). Predicting academic achievement with cognitive ability. Intelligence, 35(1), 83-92. 
Rosário, P., \& Almeida, L. (1999). As estratégias de aprendizagem nas diferentes abordagens ao estudo: Uma investigação com alunos do Ensino Secundário. Revista Galego-Portuguesa de Psicoloxia e Educación, 3(4), 273-280.

Salisbury, J., Rees, G., \& Gorard, S. (1999). Accounting for the differential attainment of boys and girls at school. School Leadership \& Management, 19, 403-427.

Sammons, P. (1995). Gender, ethnic and socio economic differences in attainment and progress: A longitudinal analysis of student achievement over nine years. British Educational Research Journal, 21(4), 465-485.

Sappington, J., \& Topolski, R. (2005). Maths performance as a function of sex, laterality, and age of pubertal onset. Laterality, 10(4), 369-379.

Senos, J. (1997). Identidade social, auto-estima e resultados escolares. Análise Psicológica, 1(XV), 123-137.

Simões, M. M. R. (2000). Investigação no âmbito da aferição nacional das matrizes progressivas coloridas de Raven (M.P.C.R.). Lisboa: Fundação Calouste Gulbenkian/Fundação para a Ciência e Tecnologia/Ministério da Ciência e da Tecnologia.

Sternberg, R., Grigorenko, E. L., \& Bundy, D. A. (2001). The predictive value of IQ. Merril-Palmer Quarterly, 47, 1-41.

Stewart, D. W., \& Louisa, V. (2006). Intelligence, academic achievement, and personality: A canonical variate analysis. Psychology in the Schools, 13(4), 468-470.

Stobart, G. Elwood, J., \& Quinlan, M. (1992). Gender bias in examination: How equal are the opportunities? British Educational Research Journal, 18(3), 261-276.

Tanner, J. M. (1962). Growth at adolescence (1 ${ }^{\mathrm{a}}$ ed.). Oxford: Blackwell.

Tanner, J. M., Healy, M. J. R., Goldstein, H., \& Cameron, N. (2001). Assessment of skeletal maturity and prediction of the adult height (TW3 method). London: W.B. Saunders.

Te Nijenhuis, J., Tolboom, E., \& Bleichrodt, N. (2004). Does cultural background influence the intellectual performance of children from immigrant groups? The RAKIT intelligence test for immigrant children. European Journal of Psychological Assessment, 20, 10-26.

Thomas, J., \& Stockton, C. (2009). Socioeconomic status, race, gender, \& retention: Impact on student achievement. Disponível em http://www.usca.edu/essays/ vol72003/stockton.pdf

Undheim, J. O., \& Nordvik, H. (1992). Socio-economic factors and sex differences in an egalitarian educational system: Academic achievement in 16-year-old Norwegian students. Scandinavian Journal of Educational Research, 36, pp. 87-98.

Waber, D. P., Mann, M. B., Merola, J., \& Moylan, P. M. (1985). Physical maturation rate and cognitive performance in early adolescence: A longitudinal examination. Developmental Psychology, 21, 666-681.

Watkins, M. W., Lei, P., \& Canivez, G. L. (2007). Psychometric intelligence and achievement: A cross-lagged panel analysis. Intelligence, 35, 59-68.

Weinstein, G. (1998). Family and intergenerational literacy in multilingual communities. Washington: National Clearinghouse for ESL Literacy Education. 


\section{MATURITY, ACADEMIC PERFORMANCE, REASONING ABILITY AND SOCIO- ECONOMIC STATUS IN 6 AND 13 YEARS OLD CHILDREN FROM LISBON}

\section{Abstract}

This essay intends to evaluate the association between maturity, academic performance (AP), reasoning ability (RA) and socio-economic status (SES) in a sample of 792 children (394 boys and 398 girls), with ages between 6 and 13 years old. Maturity was estimated through the bone age (Tanner et al., 2001) and the academic performance was obtained through inquiries to teachers, results of school tests and the levels achieved during the second term. The socio-economical status (SES) was estimated through the Graffar Scale (Graffar, 1958) and the reasoning ability (RA) by using the test of Raven's Coloured Progressive Matrices (Raven, 1963). These data were handled with the program SPSS 17.0 ( $p \leq .05)$. We verified that the AP was directly related to the SES, that the boys' RA was higher than the girls', that there were no differences between the boys' and the girls' AP on different SES classes, that the boys who were more mature obtained higher academic results than those who were less mature, that maturity was associated to the $\mathrm{AP}$, and that this association was, in general, dependent on the association between the SES and the AP.

Keywords

Maturity; Academic performance; Reasoning ability; Socio-economic status

\section{MATURITÉ, RENDEMENT SCOLAIRE, CAPACITÉ DE RAISONNEMENT ET STATUT SOCIO-ÉCONOMIQUE CHEZ LES ENFANTS DE LISBONNE ENTRE 6 E 13 ANS}

\section{Résumé}

Cette étude vise à évaluer l'association entre la maturité, le rendement scolaire (RS), la capacité de raisonnement (CR) et l'environnement socioéconomique (ESE) dans un ensemble de 792 élèves (394 garçons et 398 
filles) âgés de 6-13 ans. La maturité a été déterminée par l'âge osseux (Tanner et al., 2001) et le RS a été obtenu parmi une enquête auprès des enseignants, par les résultats des examens d'aptitude et par les niveaux atteints pendant le 2ème semestre. L'ESE a été calculé par l'Echelle de Graffar (Graffar, 1958) et la CR a été obtenue par l'application du teste de Matrices Progressives Colorées de Raven (Raven, 1963). Le traitement des données a été exécuté sur le programme SPSS 17.0 ( $\mathrm{p} \leq .05)$. Cette étude a démontré l'influence directe de l'ESE sur le RS, la supériorité de la CR des garçons sur celle des filles, qu'il n'existe pas de différence entre le RS des garçons et des filles au sein des plusieurs classes d'ESE, que les garçons plus matures obtiennent des résultats académiques supérieurs face à ceux qui sont moins matures, que la maturité est associée à la réussite scolaire et que cette association a été, dans la plupart des cas, dépendent de l'association entre l'ESE et le RS.

Mots-clé

Maturité; Rendement scolaire; Capacité de raisonnement; Environnement socio-économique

Recebido em Fevereiro/2010

Aceite para publicação em Janeiro/2012 